\title{
A Study of Background Signals in Terahertz Apertureless Near-Field Microscopy and Their Use for Scattering-Probe Imaging
}

by

Victoria Astley

A THESIS SUBMITTED

IN PARTIAL FULFILLMENT OF THE

REQUIREMENTS FOR THE DEGREE

\section{Master of Science}

APPROVED, THESIS COMMITTEE:

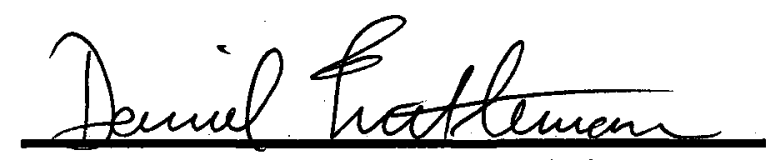

Daniel Mittleman, Professor, Chair

Electrical and Computer Engineering

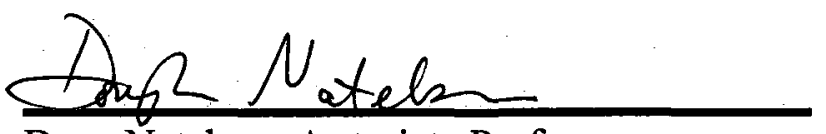

Doug Natelson, Associate Professor

Physics and Astronomy

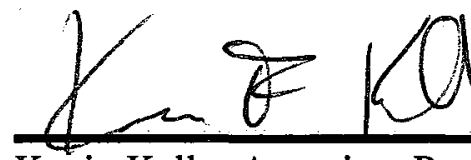

Kevin Kelly, Associate Professor

Electrical and Computer Ehgineering

HOUSTON, TEXAS

MAY 2009 
UMI Number: 1466756

\section{INFORMATION TO USERS}

The quality of this reproduction is dependent upon the quality of the copy submitted. Broken or indistinct print, colored or poor quality illustrations and photographs, print bleed-through, substandard margins, and improper alignment can adversely affect reproduction.

In the unlikely event that the author did not send a complete manuscript and there are missing pages, these will be noted. Also, if unauthorized copyright material had to be removed, a note will indicate the deletion.

\section{$\mathrm{UMI}^{\circ}$}

UMI Microform 1466756

Copyright 2009 by ProQuest LLC

All rights reserved. This microform edition is protected against unauthorized copying under Title 17, United States Code.

ProQuest LLC

789 East Eisenhower Parkway

P.O. Box 1346

Ann Arbor, Ml 48106-1346 


\section{ABSTRACT}

A Study of Background Signals in Terahertz Apertureless Near-Field Microscopy and Their Use for Scattering-Probe Imaging

by

Victoria Astley

Apertureless near-field microscopy is an imaging technique in which a small metal tip is held close to a surface, converting evanescent waves to propagating waves and permitting sub-wavelength spatial resolution. In the terahertz region of the spectrum, the interpretation of measured signals and the suppression of background scattering can be complicated by the broad bandwidth of the $\mathrm{THz}$ source and by the phase-sensitive detection of the scattered radiation. We have analyzed the use of tip-sample distance modulation for the removal of background signals. We find that significant background signals, originating from scattering off the probe tip, can be observed even after modulation. These background signals result from path-length difference modulation, and thus depend on phase-sensitive detection. We use a dipole antenna model to explain the spatial variation of this signal. Since it originates from the tip only, it can be used to characterize free-space terahertz wave fronts with sub-wavelength resolution. 


\section{Acknowledgements}

I would like to thank my advisor, Dr. Daniel Mittleman, for all his support and for believing that a weird spike in my data actually meant something. I would like to thank Dr. Rajind Mendis for allowing me to convince him that the weird spike meant something, and for all his help on the data analysis and theoretical work that followed. Additional thanks are due to Hui Zhan, for her invaluable help with the experimental setup, and to the members of my thesis committee Dr. Doug Natelson and Dr. Kevin Kelly for their time and interest. And of course, my family, for their love, support, and genetic tendency to stubbornness. 


\section{Contents}

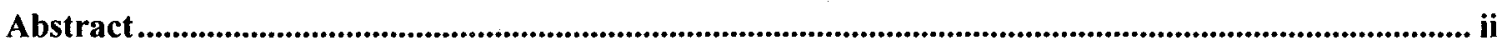

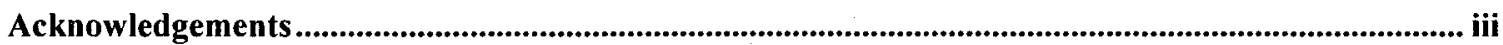

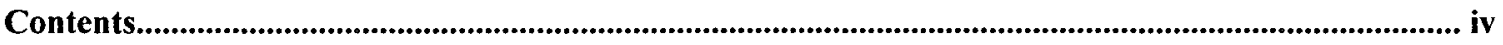

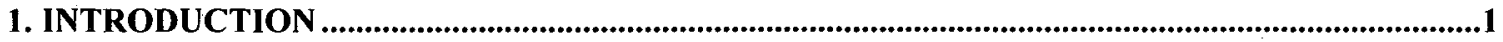

2. DETECTION OF MODULATED BACKGROUND SIGNAL ..........................................................6

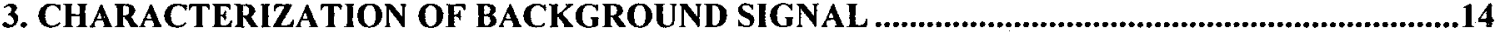

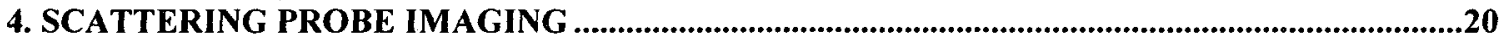

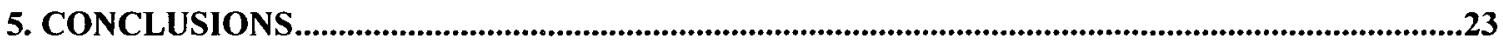

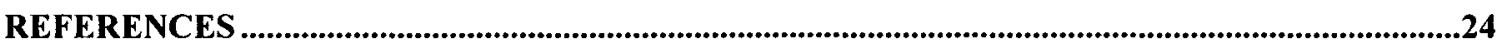




\section{INTRODUCTION}

The terahertz $(\mathrm{THz})$ region of the electromagnetic spectrum has shown great promise for a variety of spectroscopic and imaging applications. For imaging, a significant drawback has been the limited spatial resolution that can be obtained with conventional far-field techniques. To circumvent this problem, a number of near-field imaging techniques have been employed to obtain sub-wavelength-resolution $\mathrm{THz}$ images, via the use of apertures and aperture-type probes[1-7]. These either confine the illuminating radiation or limit the collection area to increase resolution, at the expense of a rapidly diminishing dynamic range in the measured signals. The implementation of apertureless near-field scanning optical microscopy (ANSOM, also called scattering or stype microscopy) in the $\mathrm{THz}$ region provides a superior method for sub-wavelength imaging. Several implementations of THz ANSOM have produced sub-wavelengthresolution images[8-11]. In a few cases, the broadband nature of the typical THz timedomain spectrometer has been used to perform spectroscopy in combination with imaging[12-16].

ANSOM was pioneered in the 1990 's as a way to obtain better resolution through the use of a scatterer rather than an aperture $[17,18]$. Whereas an aperture introduces a high wavelength-dependent transmission loss, a small scatterer such as a sphere or probe tip can actually enhance optical fields in its immediate neighborhood, or near-field. If a sample is placed in this region, the near fields are modified, and the scattered light carries information about the sample's local optical properties[17]. In ANSOM experimental implementations, the scatterer is generally a sharp metallic tip placed near the sample and illuminated with a radiation source. The light is scattered off the tip-sample junction and 
detected with external optics[19]. The spatial resolution of the image formed by rasterscanning the tip over the sample is determined by the radius of curvature of the tip apex, its scattering efficiency, and the proximity to the sample surface, rather than by the wavelength of the incident light. ANSOM experiments have been conducted in a number of spectral regions, including the visible[20], microwave[21], radio[22], and midinfrared[23], with reported resolutions ranging from $\lambda / 100$ to $\lambda / 10^{6}$.

The origin of these ANSOM signals is understood through a simple electrostatic model, in which the scatterer/probe tip is modeled as a polarizable sphere. The shape and length of the entire probe strongly affect the absolute scattering efficiency, but most ANSOM applications are concerned with the relative scattering efficiency at different locations on the sample. These are dominated by the very tip of the probe and thus the model can be simplified to include only a sphere with the same radius of curvature as the probe tip to represent the probe. The existence of the entire probe does however require a parallel polarization of the incident electric field $E_{i}$ for maximum near-field enhancement. This incident electric field polarizes the sphere, which in turn polarizes the dielectric half-space of the sample, creating an image dipole within the sample. The interaction of the original tip dipole with this image yields an effective polarizability for the system, which incorporates the local optical properties of the sample. Since the scattered field $E_{s}$ is proportional to the incident field and the effective polarizability, this implies that the scattered radiation detected in the far-field contains near-field information from the sub-wavelength-sized region directly below the tip [17, 20, 24-26].

A natural consequence of this configuration is the existence of a large background signal, since scattering will occur from both the probe tip and from the probe shaft, as 
well as from illuminated regions of the sample surface far from the tip. In order to increase signal-to-noise and to isolate the near-field signal from this considerable background, most ANSOM experiments rely on the modulation of the tip-sample separation. In the model described above, the effective polarizability depends strongly and non-linearly on the tip-sample separation (i.e., the distance between the dipole and its image), while the background scattering does not. Thus, by modulating the tip-sample separation and using lock-in detection, the tip-scattering contribution and thus the nearfield information can be distinguished from the large background [24, 27].

Techniques have been developed to optimize the modulation scheme and the extraction of the near-field signal, such as demodulation at higher harmonics $[25,28]$ and interferometric detection[26]. Most ANSOM applications involve the detection of the power intensity rather than the electric field, leading to the presence of both background and near-field signals in multiple Fourier components and a complicated analytical expression for the detected signal[29], making the extraction of a pure near-field signal challenging.

When ANSOM techniques are applied in the $\mathrm{THz}$ region, the analysis is significantly different. In the $\mathrm{THz}$ region, there are two primary differences from most other ANSOM implementations: broadband illumination and phase-sensitive detection. Due to the broadband nature of $\mathrm{THz}$ time-domain sources, the frequency-dependence of the probe response and of the spatial resolution becomes a factor. Also, a typical THz time-domain spectroscopy (THz-TDS) system permits measurement of the time-domain waveform of the electric field, rather than merely the time-averaged intensity. One consequence is that $\mathrm{THz}$ ANSOM analysis does not generally employ the polarizable- 
sphere model described above. Instead, an antenna model is used, in which the resulting ANSOM signal is proportional to the time-integral of the incident electric field $[16,30]$

In $\mathrm{THz}$ applications, modulation of the tip-sample separation is also employed for background suppression, but since the THz-TDS system detects electric field rather than intensity, the analysis is significantly less complex than for intensity-detection schemes. In the $\mathrm{THz}$ community, it has generally been assumed that the near-field and background electric fields can be separated easily at the modulation frequency, with no additional analysis. This is a significant advantage and allows most THz-ANSOM applications to treat post-lock-in signals as pure near-field information.

This assumption may not always be valid, however. Modulation of the tip-sample distance is in effect a modulation of the needle tip location, which can be a source of scattered radiation independent of the sample and a contributor to the background. For non-phase sensitive applications this is irrelevant, but since THz-TDS is phase-sensitive, modulation of the needle tip position is effectively a modulation of the phase of the scattered signal originating from the needle tip. This phase modulation is associated with the tip motion and will therefore not be filtered out by lock-in detection. The resulting signal contribution is proportional to the time-derivative of the scattered radiation from the tip. In this case, a background scattering from the needle tip, independent of the presence of the sample, may not be suppressed even with lock-in detection.

In this paper, we characterize both the near-field signals and the scattering background for the particular case of broadband $\mathrm{THz}$ radiation with coherent detection. We show that in this case, this modulated background may be present in detected ANSOM signals. We also characterize the spatial distribution of this scattering signal 
with respect to the needle, and investigate its potential as a new method for characterizing $\mathrm{THz}$ wavefronts with sub-wavelength resolution, which we name here as scatteringprobe-imaging (SPI). 


\section{DETECTION OF MODULATED BACKGROUND SIGNAL}

To investigate the scattering background described in Chapter 1, we use a THzTDS system as illustrated in Figure 1. A Ti:Sapphire laser producing $80 \mathrm{fs}$ pulses with a repetition rate of $80 \mathrm{MHz}$ is fiber-coupled to photoconductive antennas of GaAs (transmitter) and low-temperature-grown GaAs (receiver). A delay rail is used to map the time dependence of the broadband $\mathrm{THz}$ radiation. The $\mathrm{THz}$ radiation generated by the transmitter is focused using bulk polyethylene lenses onto the probe tip, a $10 \mu \mathrm{m}$ radius beryllium-copper needle at an angle of $45^{\circ}$ relative to the shaft of the probe. A polished copper substrate is placed beneath the needle to be used as a reference sample. The receiver is directed at the probe tip also at angle of $45^{\circ}$, but is placed $90^{\circ}$ out of plane (not shown in the figure). This minimizes the direct detection of the radiation that is specularly reflected from the sample.

A piezoelectric transducer is used to modulate the tip position with a frequency of $160 \mathrm{~Hz}$ and an amplitude of $0.75 \mu \mathrm{m}$ and a lock-in amplifier filters the detected radiation

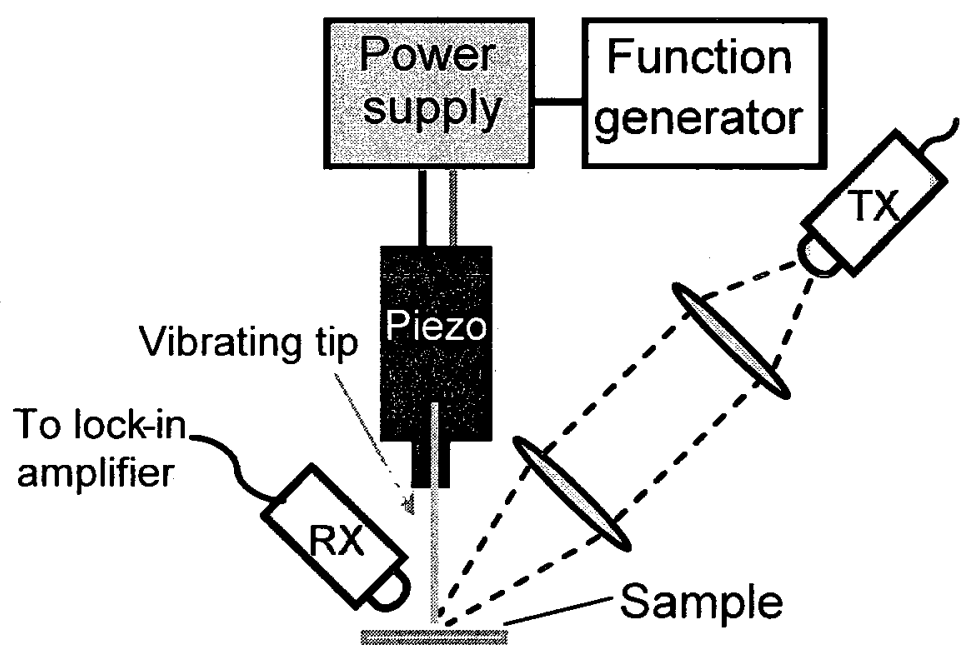

Figure 1 A schematic of the experimental setup for terahertz ANSOM. 
using the tip position as a reference. A capacitive sensor is used to determine the tip location relative to the sample surface and a proportional-integral-derivative feedback loop is used to stabilize the tip position. The electronics system used in this setup was a combination of commercially available components such as the piezotransducer and capacitive sensor, with their associated control electronics, and a homebuilt circuit board. The power supply in Figure 1 provides a DC voltage - used to control the tip-sample separation - while the function generator provides the $\mathrm{AC}$ modulation of the tip position. These two voltages are added with a summing operational amplifier, providing the setpoint for the PID feedback loop. This setpoint is compared to the output reading from the capacitive sensor and this difference is what is minimized in the feedback loop. The output of the feedback loop is fed into the piezo electronics as the control voltage for the piezotransducer. The gain of various circuit components, such as the proportional, integral, and derivative feedback mechanisms, is set (in combination with the intrinsic gain of the commercial electronic components) to obtain maximum accuracy and stability of the tip position. However, since the tip position is difficult to establish relative to the capacitive sensor, this setup provides only relative distance information, not absolute tipsample separation, so distances are given relative to the point of closest approach.

Initially, the probe tip is placed in the THz beam with no sample (substrate) present. This, combined with the out-of-plane receiver position, eliminates all potential signal sources such as direct detection or reflection from the sample surface, leaving scattering from the tip as the sole possible source of signal. Without the tip, as expected, there is no signal. With the tip in place and stationary (i.e., not position-modulated), a signal is detected. This signal is obtained by direct averaging of the photocurrent using a 
rapid-scanning delay line, and therefore is a direct measurement of the beam scattering off the probe. We refer to this as the scattered signal (see Fig. 2(a)). With this configuration, conventional descriptions of ANSOM would predict zero signal if the tip position was modulated, since there is no substrate to produce an image charge and thus no signal-modulation. However, when the tip position is modulated, and the lock-in amplifier is referenced to this tip-position modulation, a signal remains (Fig. 2(c)). This indicates a departure from the traditional ANSOM model.

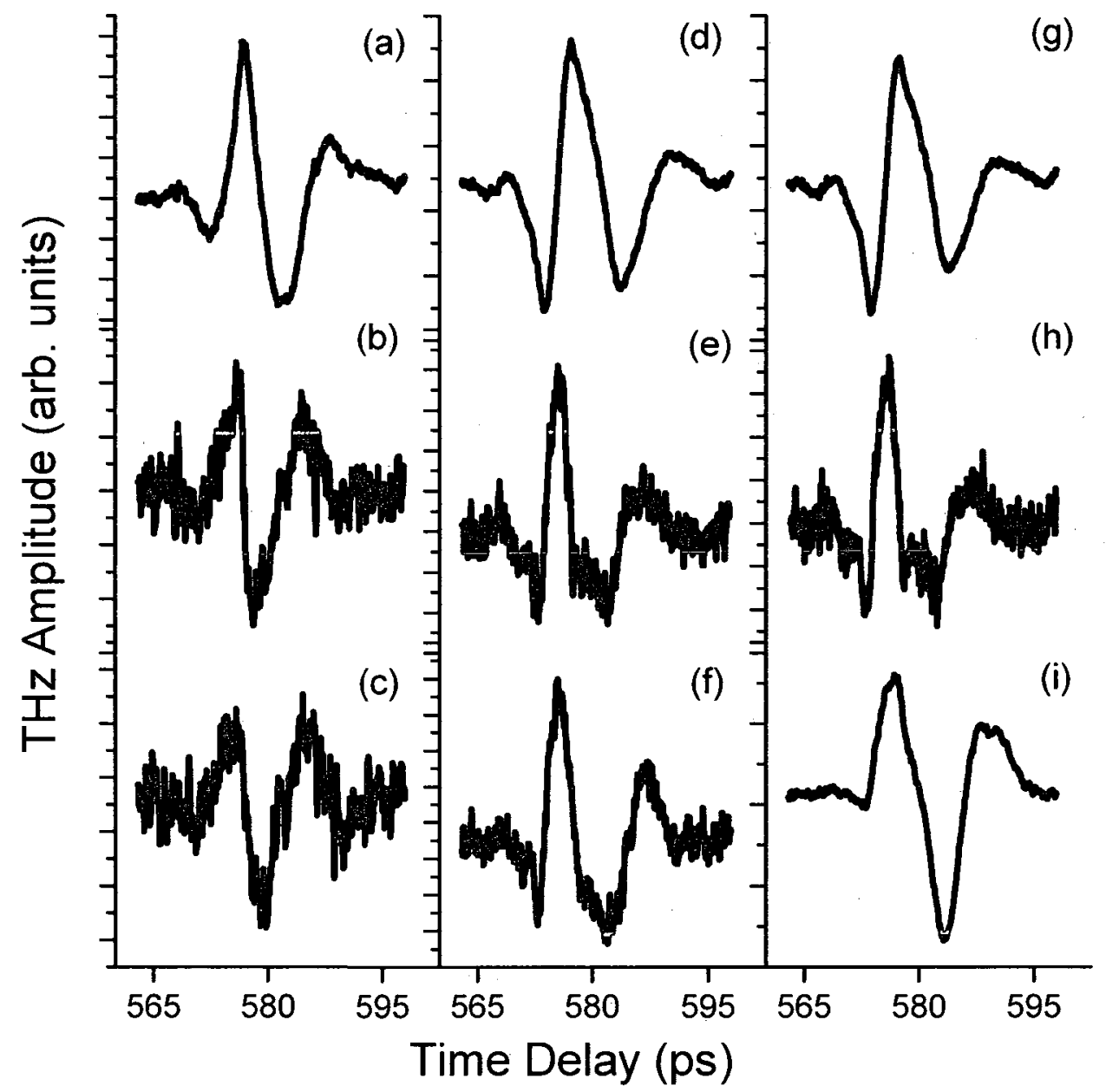

Figure 2 Terahertz time domain waveforms with no copper substrate $(\mathbf{a}, \mathrm{b}, \mathrm{c})$, copper substrate at $20 \mu \mathrm{m}(\mathrm{d}, \mathrm{e}, \mathrm{f})$, and copper substrate at $2 \mu \mathrm{m}(\mathrm{g}, \mathrm{h}, \mathrm{i})$. The top curve in each column $(\mathrm{a}, \mathrm{d}, \mathrm{g})$ is the signal with no modulation of the position of the probe tip, measured using a rapid-scanning delay line. The second curve in each column $(b, e, h)$ is the calculated time derivative of the unmodulated signal shown in the top curve. The bottom curve is the signal measured using the probe tip modulation with lock-in detection, as in a conventional ANSOM configuration $(c, f, i)$. 
Our proposed mechanism posits a phase shift caused by the motion of the tip, which modulates the path length traveled by the scattered signal. In this case, if the modulation is small compared to the wavelength, then the measured signal should be proportional to the time-derivative of the scattered $\mathrm{THz}$ waveform. To test this, the timederivative of the scattered signal (Fig. 2(a)) is calculated and presented in Fig. 2(b). Comparing this signal to Fig 2(c) yields an obvious correspondence, with all major signal features in clear agreement. This suggests that the proposed mechanism can contribute to measured THz ANSOM signals.

In a second set of measurements, a copper substrate is added at $d \sim 20 \mu \mathrm{m}$ from the tip. This is similar to a conventional ANSOM measurement, but at this large value of the tip-sample distance no near-field signal is expected[14]. Without modulation, a scattered signal from the tip is again detected (Fig. 2(d)). There is some change to the shape of the signal due to the close proximity $(d<<\lambda)$ of the copper substrate. The time-derivative of this signal is calculated as before (Fig. 2(e)) in accordance with our predicted mechanism. When the tip position is modulated, a signal again remains (Fig. 2(f)), and when compared to the calculated time-derivative there is again excellent correspondence between the two. Not surprisingly, the proposed scattering mechanism still contributes to the measured signal when the substrate is nearby. The substrate modifies the shape of the time-domain waveform, but the mechanism by which the signal is generated remains the same.

In a final measurement, the copper substrate is brought into the near-field regime, to approximately $d \sim 2 \mu \mathrm{m}$ tip-sample separation. This change from $d \sim 20 \mu \mathrm{m}$ to $d \sim$ $2 \mu \mathrm{m}$ is much less than the THz wavelength, and so it has almost no effect on the 
scattered signal measured without tip-sample modulation. A sample waveform is presented in Fig. 2(g), and it is clear that Fig. 2(g) and Fig. 2(d) are almost identical. Thus the time-derivative of the scattered signal (Fig. 2(h)) is also essentially unchanged (compare to Fig 2(e)). However, when the tip position at $d \sim 2 \mu \mathrm{m}$ is modulated (Fig. 2(i)), the signal is clearly very different, substantially larger, and no longer similar to the time-derivative. Since the tip is now within a few microns of the surface, this is evidence that the near-field component has now become comparable to (or larger than) the scattered signal component.

In the near-field regime, the antenna model described earlier[16, 30] predicts that the near-field signal is approximately proportional to the time-integral of the incident electric field. This calculated time-integral signal is shown in Figure 3(a). While it reproduces the strong negative peak of the modulated $2 \mu \mathrm{m}$ signal in Fig. 2(i), it does not reproduce the initial positive peak. This indicates that the near-field contribution cannot account for all of the features of the detected signal after modulation, at this tip-sample separation. This is in contrast to the scattered signal detected at larger tip-sample separations for which a good match is found to the initial positive peak of the signal. This suggests that the measured signal at small separations is a superposition of the nearfield component and the background signal that results from the distance modulation effect.

When the calculated time-integral of the incident THz field (Fig 3(a) is added to the far-field scattered signal after modulation (Fig 2(f)), a superposition which is qualitatively resembles the experimental waveform can be obtained (Fig. 3(b)), reproducing the major features such as the two positive peaks and single negative peak. 


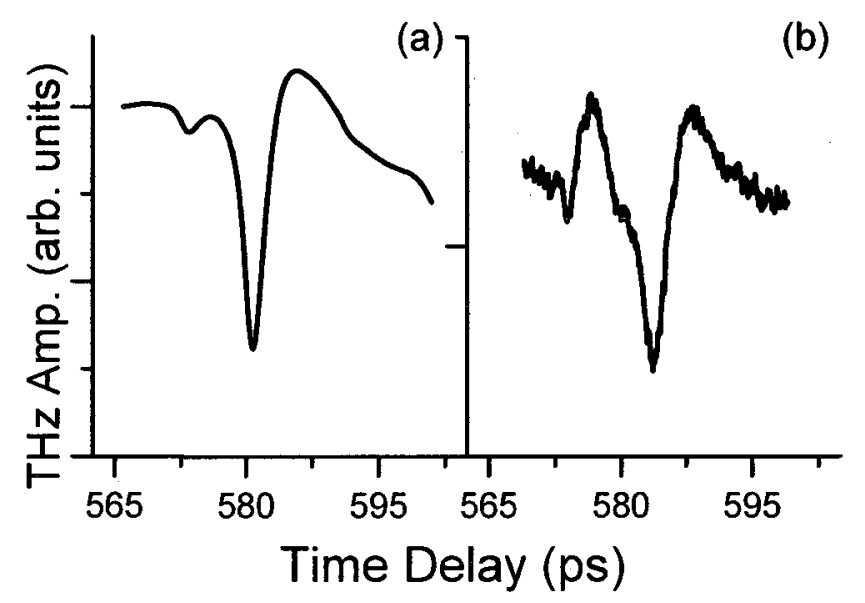

Figure 3 A diagram of the superposition process. The time integral of the incident electric field (a) and the background scattered signal (from Fig. 2(f)) are added with a scaling factor to obtain the superposition of the two (c).

This suggests that the detected ANSOM signal in the near-field regime is composed of both the near-field signal, which contains information about the substrate, and a background signal originating from tip scattering, which does not.

The superposition of the waveforms shown in Figure 3(b) was generated with an arbitrary scaling factor relating the two components. The exact value of this scaling factor is uninteresting, since it depends on the gain in various electronics in the system such as the lock-in amplifier. However, the relationship of this scaling factor to the tipsample distance is interesting. Since the near-field contribution is the primary source of the negative peak and the background contribution the source of the initial positive peak of the detected signal, comparison of these two amplitudes as a function of tip-sample separation $d$ gives a rough measure of how the two distinct signal contributions scale with distance (see Fig. 4). At high tip-sample separations, the near-field contribution is essentially zero as expected, while the background is constant, independent of $d$. As the 
separation decreases, both contributions increase significantly, with the near-field contribution showing a steeper dependence on distance.

These results confirm that a substantial background scattering signal may remain after modulation of the tip position, and also support phase-modulation as the mechanism. This is significant for ANSOM applications where this background may be an issue and must be taken into account in signal analysis. Unfortunately, Fig.4 demonstrates that a simple reference scan and subtraction is unlikely to be sufficient, as the background depends sensitively on $d$ in the near field regime.

This background does not necessarily appear in all ANSOM experimental configurations. Its amplitude depends on the modulation amplitude and the tip diameter, along with receiver position and the orientation of the tip in the beam. That said, it cannot be ignored as an experimental possibility, particularly when using a broadband THz source. The near-field signal is naturally low-bandwidth due to the approximate $1 / \omega$ filtering due to antenna effects[16, 30]. In contrast, the background signal is a timederivative, meaning its bandwidth can be considerably larger. In the initial discovery of this background, it was observed because of an unexpected sharp high-bandwidth peak superimposed on a previously low-bandwidth near-field $\mathrm{THz}$ waveform. This additional bandwidth does not necessarily contain any near-field information and so can produce erroneous results if it is mistaken for part of the near-field signal. 


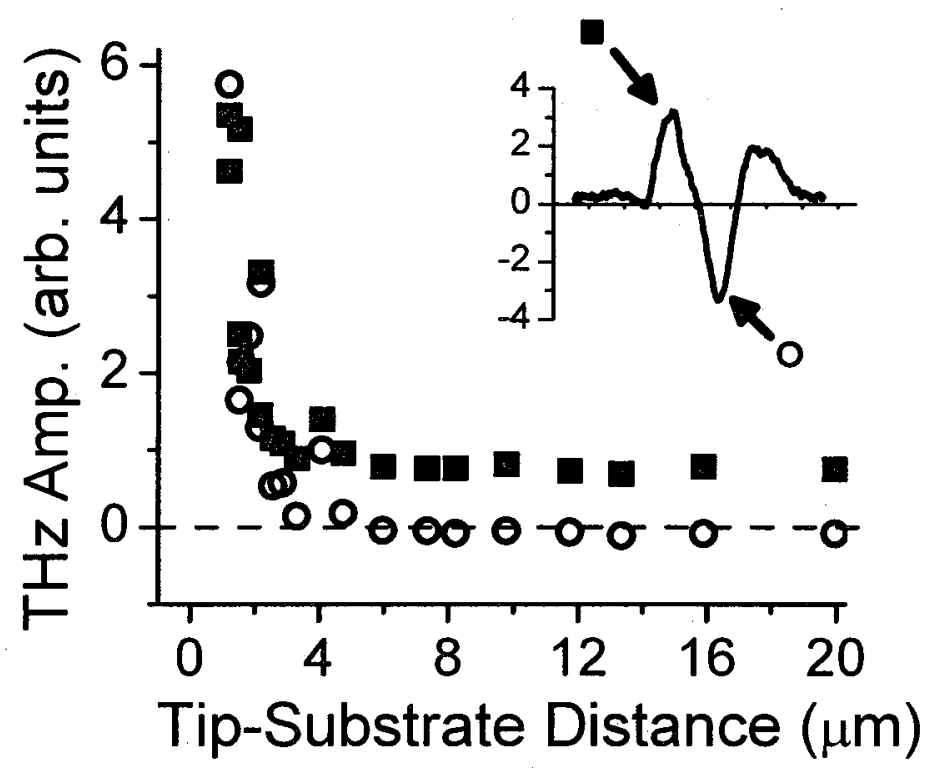

Figure 4 Approach curves showing the distance dependence of the positive (square) and negative (circle) peaks of the THz ANSOM signal.

Another consequence of this persistent background signal is the possibility of exploiting it for measuring free-space $\mathrm{THz}$ wavefronts with sub-wavelength resolution that is, ANSOM with no substrate. It could be used to characterize $\mathrm{THz}$ spatial fields such as those emitted by a photoconductive antenna, transmitted through metamaterials, or radiated from the end of a waveguide. Such characterization could be performed with sub-wavelength resolution because the scattered signal originates only from a small, subwavelength-sized metal tip immersed in the beam that one wishes to examine. With an eye towards establishing the potential of this technique, we first characterized the spatial pattern of the scattered radiation from the tip and then conducted a proof-of-concept test on a free-space Gaussian beam. 


\section{CHARACTERIZATION OF BACKGROUND SIGNAL}

For the characterization of the radiation patterns scattered from the probe tip, we removed the substrate and placed the tip directly at the focus of an incident free-space $\mathrm{THz}$ beam, still with a $45^{\circ}$ angle of incidence, as illustrated in Figure 5(a). With no substrate, the tip position was maintained by a feedback loop based on an internal strain gauge of the piezotransducer, rather than an external capacitive sensor. The modulation amplitude was increased to $10 \mu \mathrm{m}$ (still much less than the $\mathrm{THz}$ wavelength) in order to increase the amplitude of the measured signal.

The receiver was placed on a rotating stage approximately $6 \mathrm{~mm}$ away from the tip in two different configurations. In the H-plane configuration, the dipole axis of the receiver is perpendicular to the axis of the tip and the axis of the tip is the axis of rotation. The location behind the tip, opposite from the direction of incident radiation, is defined as $180^{\circ}$ and the location directly in front, obstructing the incident radiation, is $0^{\circ}$ (see Fig. 5(a)). In the E-plane configuration, the dipole axis of the receiver is aligned with the axis of the tip and rotates around the tip, obtaining signals at positions to the sides and below

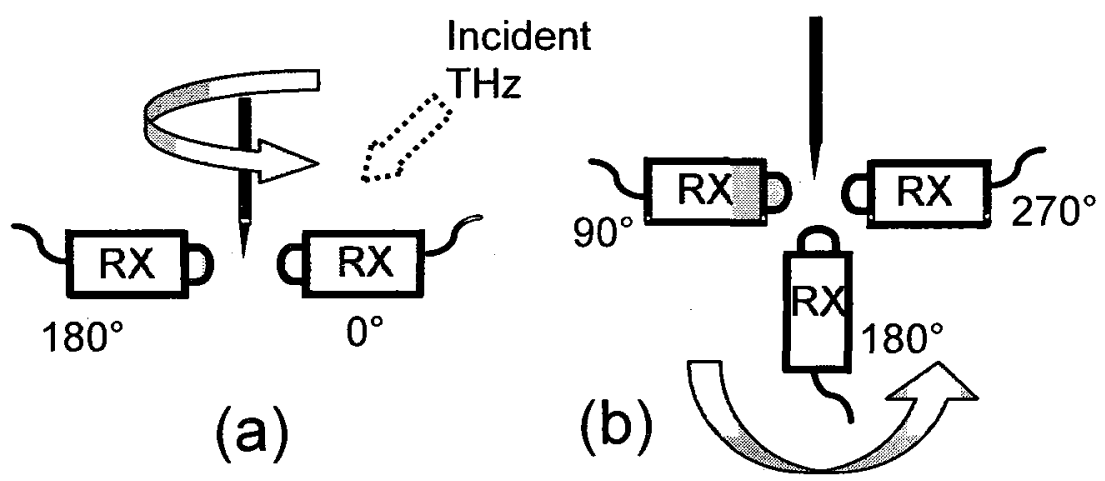

Figure 5 Schematic of the experimental setup for the measurement of the spatial radiation pattern. (a) The H-plane configuration, in which the axis of rotation of the receiver is parallel to the probe tip axis. (b) The E-plane configuration, in which the axis of rotation is perpendicular to the probe tip axis. In the E-plane configuration pictured, $\mathrm{THz}$ radiation is incident at a $45^{\circ}$ angle onto the tip in a direction pointing into the page. 
the tip while pointing directly at the tip at all locations (as shown in Fig 5(b)). The point directly below the tip, pointing upward, is defined as $180^{\circ}$.

The data obtained from these two configurations are given in Figure 6 . In the $\mathrm{H}$ plane (Fig. 6(a)), the radiation appears nearly isotropic, with no variation as the receiver is rotated around the tip. In the E-plane (Fig. 6(b)), the signal pattern is clearly more complicated, exhibiting a minimum directly beneath the needle, but also with secondary minima located roughly at the intersection with the H-plane (at $90^{\circ}$ and $270^{\circ}$ )

In a simple dipole antenna model, the radiation pattern in 3-D space is toroidal, isotropic in the H-plane and with a double-lobed pattern in the E-plane. The data clearly shows a nearly isotropic pattern in the H-plane as expected for a dipole, but the E-plane pattern does not match. A simple dipole model predicts a minimum directly beneath the tip, but maxima at the intersection with the H-plane where the data show relative minima.

To understand this, we develop a model based on the half-wave dipole and incorporate the spatial pattern of the path length difference as seen by the receiver. The

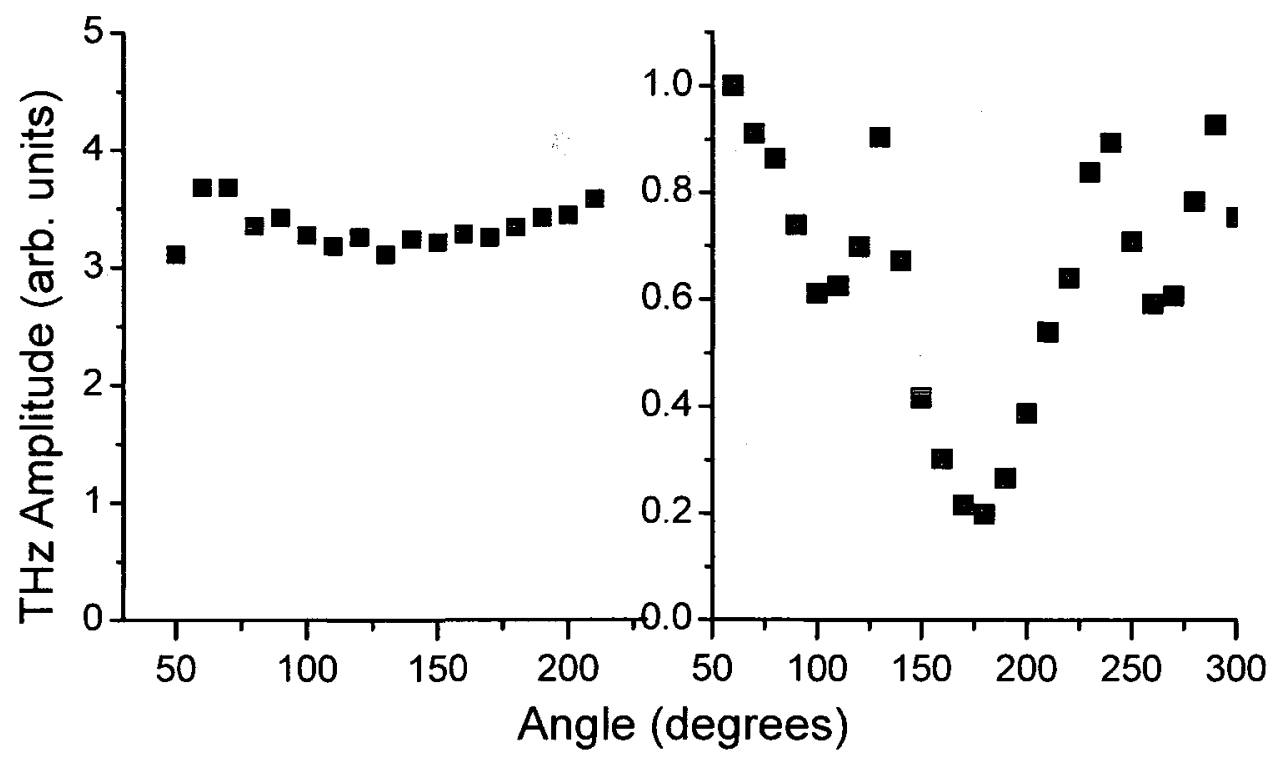

Figure 6 H-plane (a) and E-plane (b) radiation patterns. The squares mark the experimental data. 
half-wave dipole electric field amplitude at a particular distance from the needle is given by[31]:

$$
\mathrm{A}(\theta)=\frac{\cos (k l \cos (\theta))-\cos (k l)}{\sin (\theta)}
$$

where $k$ is the wave vector $(2 \pi / \lambda), l$ is the length of the antenna (in this case $\lambda / 2$ for a half-wave dipole), and $\theta$ is the angle as defined in Figure 5(b). This equation yields a toroidal pattern as described above.

Figure 7 (a) shows this spatial pattern both as a diagram and as a curve plotted versus angle $\theta$ for comparison to the experimental data. While the dipole pattern reproduces the minimum below the needle, it does not adequately describe other features of the experimental data.

The half-wave dipole pattern in Eq. 1 describes a situation in which the detection efficiency is independent of receiver position, and the radiated signal is detected identically at all points. In our experimental case, the strength of the detected signal also depends on the geometry of the setup. The mechanism for the generation of this signal is the path-length difference created as the tip position is modulated. That path length difference is clearly dependent on the relative position of the receiver - if the receiver is located exactly perpendicular to the direction of modulation, the motion is parallel to the direction of propagation, and as such there is a minimum of path length change as the tip moves. If the receiver is located directly below the tip $\left(180^{\circ}\right)$, the motion of the tip is entirely in the direction of propagation, giving a maximum path length change. In the $\mathrm{H}$ plane, the receiver moves in a plane of constant path length change, while in the E-plane the path length change is a function of the receiver position. 
To obtain the spatial variation of this effect, the path length difference can be calculated based on the angle $\theta$ between the axis of the tip and the direction of propagation (also defined as $180^{\circ}$ with the receiver below the needle, as in Fig. $5(\mathrm{~b})$ ), as well as the distance to the receiver $R$ and the amplitude of modulation . The path length difference as the needle moves, , is given by:

$$
\Delta L(\theta)=\sqrt{R^{2}+2 R \cos (\theta) \Delta x+\Delta x^{2}}-R
$$

To match the experimental parameters, we set $R=6 \mathrm{~mm}$ and $\quad=10 \mu \mathrm{m}$.

Figure 7(b) shows the diagram of this pattern relative to the needle and the comparison to the experimental data. The path length difference pattern does not agree adequately with the experimental pattern, but it does produce the minima at the H-plane that are absent in the dipole pattern of Equation 1. Since the output from the lock-in is not based entirely on either the dipole emission pattern of the needle or the path length difference pattern observed by the receiver, a combination of these two patterns was generated.

The output from the lock-in amplifier is the difference between the maximum and minimum values of the modulated signal from the receiver. So as the tip moves, the receiver output varies from the signal at maximum path length difference to minimum path length difference. Assuming that the dipole radiation pattern $A(\theta)$ remains unchanged over the $10 \mu \mathrm{m}$ range, the output from the receiver is therefore proportional to the product of $A(\theta)$ and the propagation term, $\cos (k d)$. The signal output from the lock-in amplifier is therefore given by:

$$
\Delta V(\theta)=A(\theta) *(1-\cos (k \Delta L(\theta))
$$


This curve is given as a diagram and plotted with the experimental data in Figure 7(c). Since it combines the various features from the dipole pattern (i.e. the minimum at $180^{\circ}$ ) and from the path length difference dependence on angle (i.e. the minima at $90^{\circ}$ and $270^{\circ}$ ), this curve matches the experimental data reasonably well, reproducing all of the local maxima and minima. However, it does not accurately reproduce the difference in signal strength at the primary and secondary minima. In order to account for this, the finite size of the collecting aperture of the $\mathrm{THz}$ receiver must be considered.

Since the $\mathrm{THz}$ receiver is placed very close to the metal tip, it collects radiation over some large solid angle, which depends on the distance of the receiver from the tip antenna. At a distance of $6 \mathrm{~mm}$, with an estimated receiver aperture diameter of $6 \mathrm{~mm}$, this corresponds to an angle of integration of approximately $60^{\circ}$. When the $\theta$ ) pattern is averaged over a $60^{\circ}$ window, the resulting curve agrees very well with the experimental results, as shown by the curve in Figure 7(d). Thus the model of a halfwave dipole in combination with a path-length geometrical effect is an accurate description of the experimental observations of the E-plane radiation pattern of the tip antenna.

Although a half-wave dipole was used for simplicity, using other dipole lengths up to $3 \lambda / 2$ produced no significant change in the theoretical curve. Also, the calculations were based on a frequency of $100 \mathrm{GHz}$, but a variation of frequencies within the detected signal's bandwidth produced only slight shifts in the theoretical curve. This analysis also assumes that the tip is placed exactly at the axis of rotation of the receiver, which may explain the slight deviation in the experimental plot. 

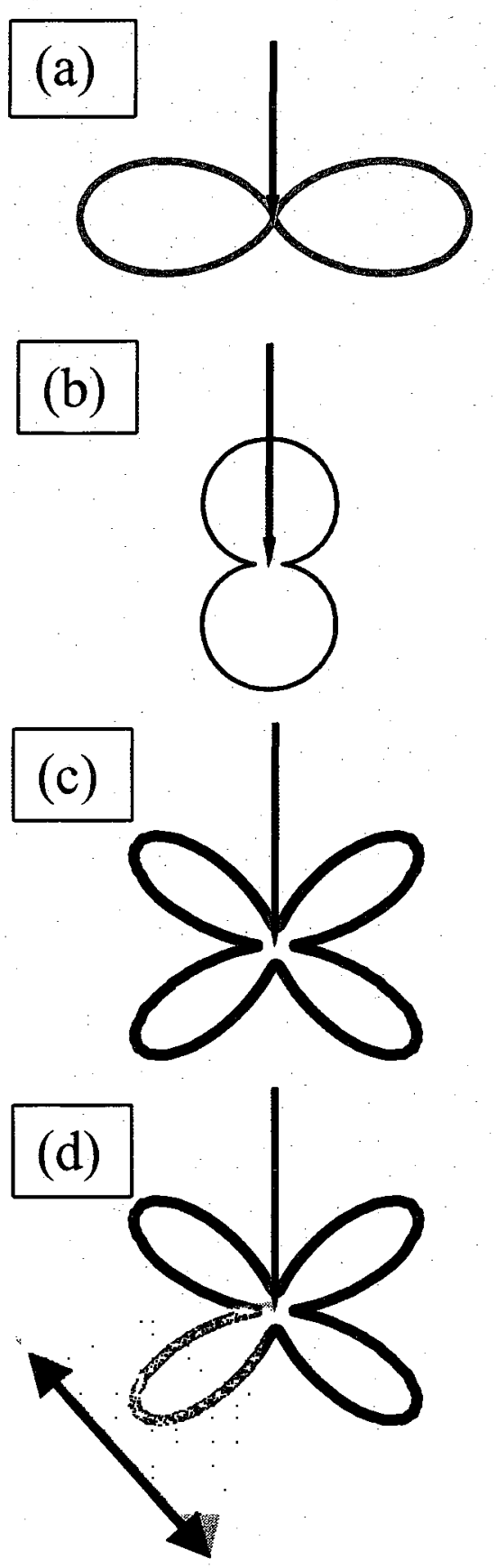
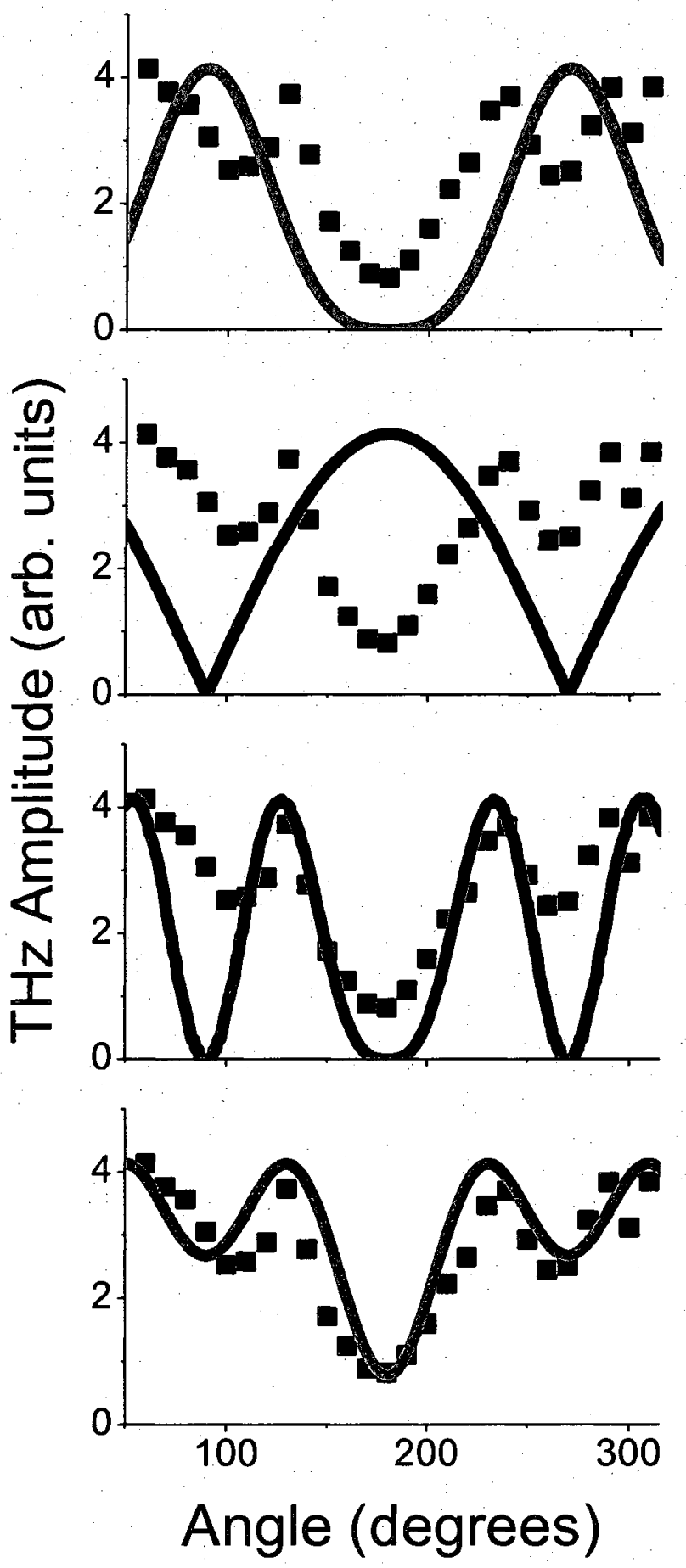

Figure 7 An illustration of the process of fitting a curve to the E-plane spatial pattern data. In all the graphs, the red squares are the experimental data (as in Figure 6(b)). In this figure, (a) shows the dipole pattern, both as a diagram and on the graph, in green. In (b), the blue lines on the graph and diagram show the path length difference pattern. In (c), the dark blue lines are combination pattern in Equation 3, while (d) shows the combination pattern after integration due to large aperture size. 


\section{SCATTERING PROBE IMAGING}

Finally, we conduct a proof of concept test for the scattering-probe imaging technique, whereby we determine the spatial profile of a free-space $\mathrm{THz}$ Gaussian beam. If this scattering signal is a measure of the amplitude of the local $\mathrm{THz}$ electric field at the probe tip, then it should be possible to use it to map a spatially distributed $\mathrm{THz}$ field, using the methods of ANSOM but without a substrate.

Using a similar experimental setup to that shown in Figure 1, the probe tip is placed at the focus of an incident Gaussian beam with its axis parallel to the electric field polarization, and its position is modulated with $\Delta x \sim 10 \mu \mathrm{m}$ amplitude (Figure 8(a)). The receiver is placed out of plane by $90^{\circ}$ and directed at the needle tip. Both the tip and receiver are mounted on a translation stage and moved in unison across the input $\mathrm{THz}$ beam profile, along a line perpendicular to both the tip and the input beam propagation direction. Without the tip in place, there is no signal detected, ensuring that the detected signal can only result from scattering off the tip.
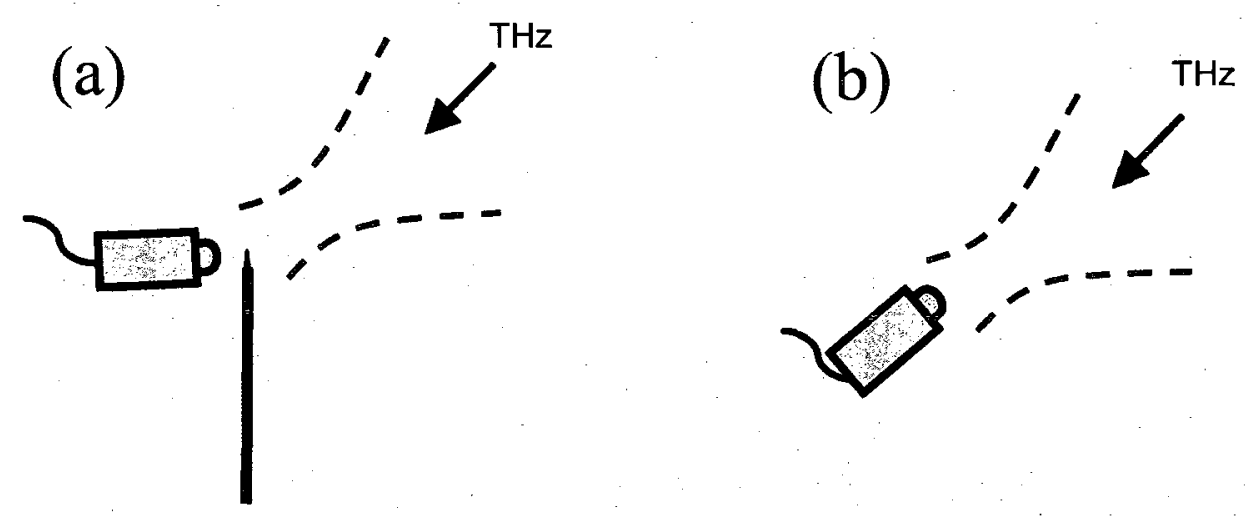

Figure 8 The experimental setup for the proof of concept test for scattering probe imaging (a) and a comparison test with the receiver directly in the beam (b). For the SPI setup in (a), the receiver was placed $90^{\circ}$ out of the plane of the paper. 


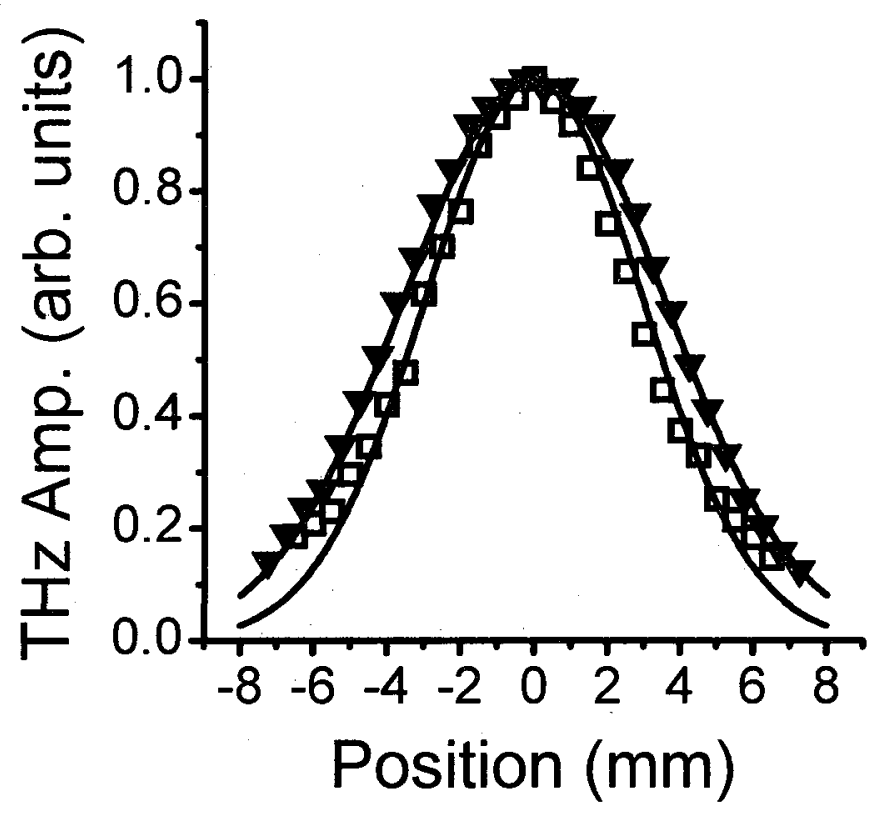

Figure 9 Gaussian beam profile measured using scattering probe imaging. The peak-topeak THz signal amplitudes are plotted for both the SPI technique (squares) and with the receiver directly in the beam (triangles). The solid lines are theoretical Gaussian curves with waist sizes of $8.4 \mathrm{~mm}$ and $10.1 \mathrm{~mm}$ respectively. All amplitudes are normalized for comparison.

With the tip in place, the peak-to-peak THz amplitude of the detected scattered signal is plotted in Figure 9 (circles). The data clearly show a Gaussian distribution of the signal, consistent with a $1 / e$ beam diameter of $8.4 \mathrm{~mm}$. We have fit a theoretical Gaussian curve with this diameter (solid curve) to the data, which shows good agreement with the shape of the pattern.

For comparison, the receiver was used to map the Gaussian beam directly, without the needle. The receiver was placed at the focus and pointed directly into the path of the beam (Fig. 8(b)). The results are quite similar to the needle-scattering data, but with a larger diameter of $10.1 \mathrm{~mm}$ (Fig. 9, squares), due to the finite aperture of the receiver.

The shape still matches a Gaussian profile. The optical arrangement for the incident beam consists of two confocally aligned lenses, with the transmitter at the focus of the 
first lens and the receiver or needle tip at the focus of the other. For a $6 \mathrm{~mm}$ aperture of the transmitter, this is predicted to give a $6 \mathrm{~mm}$ beam waist diameter to the Gaussian beam waist at the focus. Considering the rough positioning of the needle with respect to the focal plane, this is reasonable agreement. 


\section{CONCLUSIONS}

We describe an effect whereby a phase-sensitive detection method such as THzTDS may detect an additional background scattered signal from the modulation of probetip position in ANSOM. We have characterized this background signal, and shown that it can contribute substantially to measured ANSOM signals, thus possibly complicating their interpretation. This scattered signal can be used to perform spatially-resolved measurements of freely propagating $\mathrm{THz}$ fields, in a configuration we deem scattering probe imaging. It can be useful for measuring $\mathrm{THz}$ beams with high (sub-wavelength) spatial resolution, a technique that could be valuable for characterizing waveguides, metamaterials, photonic crystals, and other similar structures. 


\section{REFERENCES}

[1] S. Hunsche, M. Koch, I. Brener, and M. C. Nuss, Opt. Commun. 150, 22 (1998).

[2] O. Mitrofanov, I. Brener, M. C. Wanke, R. R. Ruel, J. D. Wynn, A. J. Bruce, and J. Federici, Appl. Phys. Lett. 77, 591 (2000).

[3] O. Mitrofanov, I. Brener, R. Harel, J. D. Wynn, L. N. Pfeiffer, K. W. West, and J. Federici, Appl. Phys. Lett. 77, 3496 (2000).

[4] Y. Kawano and K. Ishibashi, Nature Photonics 2, 618 (2008).

[5] Q. Chen, Z. Jiang, G. X. Xu, and X.-C. Zhang, Opt. Lett. 25, 1122 (2000).

[6] J. Federici, O. Mitrofanov, M. Lee, J. W. P. Hsu, I. Brener, R. Harel, J. D. Wynn, L. N. Pfeiffer, and K. W. West, Phys. Med. Biol. 47, 3727 (2002).

[7] J. Bae, T. Okamoto, T. Fujii, K. Mizuno, and T. Nozokido, Appl. Phys. Lett. 71, 3581 (1997).

[8] H.-T. Chen, R. Kersting, and G. C. Cho, Appl. Phys. Lett. 83, 3009 (2003).

[9] H. Zhan, V. Astley, M. Hvasta, J. A. Deibel, and D. M. Mittleman, Appl. Phys. Lett. 91, 162110 (2007).

[10] P. C. M. Planken and N. C. J. Van der Valk, Opt. Lett. 29, 2306 (2004).

[11] A. J. Huber, F. Keilmann, J. Wittborn, J. Aizpurua, and R. Hillenbrand, Nano. Lett. 8,3766 (2008).

[12] F. Buersgens, R. Kersting, and H.-t. Chen, Appl. Phys. Lett. 88, 112115 (2006).

[13] P. C. M. Planken, C. E. W. M. van Rijmenam, and R. N. Schouten, Semicond. Sci. Technol. 20, S121 (2005).

[14] N. C. J. Van der Valk and P. C. M. Planken, Appl. Phys. Lett. 81, 1558 (2002). 
[15] H.-G. von Ribbeck, M. Brehm, D. W. van der Weide, S. Winnerl, O. Drachenko, M. Helm, and F. Keilmann, Opt. Express 16, 3430 (2008).

[16] K. Wang, D. M. Mittleman, P. C. M. Planken, and N. C. J. Van der Valk, Appl. Phys. Lett. 85, 2715 (2004).

[17] F. Keilmann and R. Hillenbrand, Phil. Trans. R. Soc. Lond. A 362, 787 (2004).

[18] J. Wessel, J. Opt. Soc. Am. B 2, 1538 (1985).

[19] Y. Inouye and S. Kawata, Opt. Lett. 19, 159 (1994).

[20] F. Zenhausern, Y. Martin, and H. K. Wickramasinghe, Science 269, 1083 (1995).

[21] B. Knoll, F. Keilmann, A. Kramer, and R. Guckenberger, Appl. Phys. Lett. 70, 2667 (1997).

[22] F. Keilmann, D. W. van der Weide, T. Eickelkamp, R. Merz, and D. Stockle, Opt. Commun. 129, 15 (1996).

[23] A. Lahrech, R. Bachelot, P. Gleyzes, and A. C. Boccara, Opt. Lett. 21, 1315 (1996).

[24] R. Hillenbrand and F. Keilmann, Phys. Rev. Lett. 85, 3029 (2000).

[25] B. Knoll and F. Keilmann, Opt. Commun. 182, 321 (2000).

[26] R. Hillenbrand, B. Knoll, and F. Keilmann, J. Microsc. 202, 77 (2001).

[27] E. A. Ash and G. Nicholls, Nature 237, 510 (1972).

[28] P. G. Gucciardi, G. Bachelier, and M. Allegrini, J. Appl. Phys. 99, 124309 (2006).

[29] C. H. Chuang and Y. L. Lo, Opt. Express 15, 15782 (2007).

[30] G. C. Cho, H.-T. Chen, S. Kraatz, N. Karpowicz, and R. Kersting, Semicond. Sci. Technol. 20, 286 (2005).

[31] J. D. Kraus, Antennas (McGraw-Hill, New York, 1950). 